I. Satake

Nagoya Math. J.

Vol. 46 (1972), 121-145

\title{
LINEAR IMBEDDINGS OF SELF-DUAL HOMOGENEOUS CONES
}

\author{
I. SATAKE*)
}

Introduction. Let $G$ be a reductive algebraic Lie group ${ }^{1)}$ acting linearly on a (finite-dimensional) real vector-space $U$ with a maximal compact isotropy subgroup $K$ and suppose that the quotient $\Omega=G / K$ is a selfdual homogeneous cone in $U$. Let $\left(G^{\prime}, K^{\prime}\right)$ be another such pair corresponding to a self-dual homogeneous cone $\Omega^{\prime}$ in $U^{\prime}$. Given a homomorphism $\rho: G \rightarrow G^{\prime}$ such that $\rho(K) \subset K^{\prime}$, one has an equivariant map $\Omega \rightarrow \Omega^{\prime}$ induced by $\rho$, which we denote again by $\rho$. If this map is a restriction to $\Omega$ of an injective linear map of $U$ into $U^{\prime}$, it will simply be called a linear imbedding of $\Omega$ into $\Omega^{\prime}$. The purpose of this paper is to determine all homomorphisms $\rho: G \rightarrow G^{\prime}$ giving rise to a linear imbedding of $\Omega$ into $\Omega^{\prime}$ in this sense, for the case where $G^{\prime}=G L(n, \mathscr{K})(\mathscr{K}=\boldsymbol{R}, \boldsymbol{C}, \boldsymbol{K})$ (see 1.2). After a brief summary of known facts in 1 (due mainly to Koecher [2] and Vinberg [4]), we will in 2 reformulate our problem in terms of the representation-theory of Lie algebras and state the main results. Sections 3 and 6 are devoted to the reductions of the problem to the case where $\mathscr{K}=\boldsymbol{C}$ and where both $\Omega$ and $\rho$ are irreducible. The solutions for this particular case are given in 4 .

Our problem is closely related to a similar problem concerning holomorphic imbeddings of symmetric domains ([3a]). (Actually, our method here is nothing but a replica of the old one.) It will turn out (as the result of the classification) that every linear imbedding $\rho: \Omega \rightarrow \Omega^{\prime}=\mathscr{P}(n, C)$ is an isometry such that the image $\rho(\Omega)$ is totally geodesic in $\Omega^{\prime}$, and can be extended to a holomorphic imbedding of the corresponding tube domain: $U+i \Omega \rightarrow U^{\prime}+i \Omega^{\prime}$. It would be desirable to prove these facts more directly

Received September 8, 1971.

* Partially supported by NSF Grant GP-20436.

1) Here by a (reductive) algebraic Lie group we mean a Lie group whose identity connected component is that of the group of $\boldsymbol{R}$-rational points of a connected (reductive) algebraic group $\mathscr{G}$ defined over $\boldsymbol{R}$. $\mathscr{G}$ is reductive, if its radical is an (algebraic) torus. 
(without using the classification) and including possibly the case where $\Omega^{\prime}$ is not of classical type. Out study was motivated by, and in turn has some applications to, the theory of (rational) Siegel domains of the third kind, which we hope to discuss elsewhere.

\section{Self-dual homogeneous cones.}

1.1. In the following, $U$ is a real vector-space of dimension $m$ and $\Omega$ is a (non-empty) open convex cone in $U$. We suppose that the vertex of $\Omega$ is the origin of $U$ and $\Omega$ does not contain any straight line. Let $G=\operatorname{Aut}_{0}(\Omega)$ be the identity connected component of the (linear) automorphism group of $\Omega$, i.e., the subgroup of $G L(U)$ formed of all linear transformations $g$ such that $g(\Omega)=\Omega$. We shall always assume $\Omega$ to be "homogeneous" and "selfdual"; these mean, respectively, that $G$ is transitive on $\Omega$ and that there exists a positive-definite inner product $\langle>$ on $U$ such that one has

$$
\left.\omega \in \Omega \Longleftrightarrow\left\langle\omega, \omega^{\prime}\right\rangle\right\rangle 0 \text { for all } \omega^{\prime} \in \bar{\Omega}, \omega^{\prime} \neq 0 .
$$

It is known ([4]) that $G$ is then a reductive (algebraic) Lie group with the center isomorphic to a vector group and that for any $\omega_{0} \in \Omega$ the isotropy subgroup $K=K_{\omega_{0}}$ of $G$ at $\omega_{0}$ is a maximal compact subgroup of $G$. Thus $\Omega$ has a structure of a symmetric space with non-positive curvature (with a flat part) with respect to a $G$-invariant Riemannian metric. Furthermore, there exists a unique direct decomposition

$$
\Omega=\Omega_{1} \times \cdots \times \Omega_{t}, U=U_{1} \oplus \cdots \oplus U_{t}
$$

such that $\Omega_{i}$ is an irreducible (i.e., indecomposable) self-dual homogeneous cone in $U_{i}$, and correspondingly the automorphism group is also decomposed in the form:

$$
G=G_{1} \times \cdots \times G_{t}, G_{i}=\operatorname{Aut}_{0}\left(\Omega_{i}\right),
$$

where each $G_{i}$ is the direct product of a simple (semisimple) Lie group (which may reduce to the identity group) and the group of dilatations on $U_{i}$, isomorphic to $\boldsymbol{R}$.

1.2. The irreducible self-dual homogeneous cones are completely classified (up to linear isomorphisms) into the following five types ([2], [4]):

$$
\begin{aligned}
& 1^{\circ} . \quad \Omega=\mathscr{P}(\nu, \boldsymbol{R}), m=\frac{1}{2} \nu(\nu+1), \\
& 2^{\circ} . \quad \Omega=\mathscr{P}(\nu, \boldsymbol{C}), \quad m=\nu^{2},
\end{aligned}
$$


$3^{\circ} . \quad \Omega=\mathscr{P}(\nu, K), m=2 \nu^{2}-\nu$,

where, as usual, $\boldsymbol{R}, \boldsymbol{C}, \boldsymbol{K}$ denote the field of reals, complexes and quaternions, respectively, and the symbol $\mathscr{P}(\nu, \mathscr{K})(\mathscr{K}=\boldsymbol{R}, \boldsymbol{C}, \boldsymbol{K})$ stands for the cone formed of all positive hermitian elements in $M_{\nu}(\mathscr{K})$ (the ring of all $\nu \times \nu$ matrices with entries in $\mathscr{K}$ ) with respect to the standard involution $x=\left(x_{i j}\right) \longmapsto{ }^{t} \bar{x}=\left(\bar{x}_{j i}\right)$. The semisimple part $G^{S}$ of $G=\operatorname{Aut}_{0}(\Omega)$ is identified with $S L(\nu, \mathscr{K}) /$ center, if the action of $g \in S L(\nu, \mathscr{K})$ on $\mathscr{P}(\nu, \mathscr{K})$ is defined by $x \longmapsto{ }^{t} g^{-1} x g^{-1}$. $(S L(\nu, \mathscr{K})$ denotes the multiplicative group of all elements in $M_{\nu}(\mathscr{K})$ with reduced norm 1.) These three types are called "classical." Note that, for $\nu=1$, they all reduce to the "half-line" $\{x \in \boldsymbol{R} \mid x>0\}$, for which one has $G^{s}=\{1\}$.

$$
4^{\circ} . \quad \Omega=\left\{x=\left(x_{i}\right) \in \boldsymbol{R}^{m} \mid x_{1}^{2}-\sum_{i=2}^{m} x_{i}^{2}>0, x_{1}>0\right\} \quad(m \geqslant 3, m \neq 4) .
$$

$G^{s}$ is the identity connected component of $S O(1, m-1)$.

$$
5^{\circ} . \quad \Omega=\mathscr{P}(3, \mathscr{C}), m=27,
$$

where $\mathscr{C}$ denotes the Cayley's octonion algebra and $\mathscr{P}(3, \mathscr{C})$ is the cone formed of all positive elements in the 27-dimensional exceptional Jordan algebra. The semisimple part $G^{s}$ is a simple Lie group of type $E_{6}$ with real rank 2.

\section{Linear imbeddings.}

2.1. Let $\Omega$ and $\Omega^{\prime}$ be self-dual homogeneous cones in real vector-spaces $U$ and $U^{\prime}$, respectively, and let $G=\operatorname{Aut}_{0}(\Omega), G^{\prime}=\operatorname{Aut}_{0}\left(\Omega^{\prime}\right)$. An injection $\rho$ of $\Omega$ into $\Omega^{\prime}$ is called a linear imbedding if the following conditions are satisfied:

(i). $\rho$ is "equivariant", i.e., there exists a local monomorphism of $G$ (that is, a monomorphism from a covering group of $G$ ) into $G^{\prime}$, denoted again by $\rho$, such that one has

$$
\rho(g(\omega))=\rho(g)(\rho(\omega)) \quad \text { for } g \in G, \omega \in \Omega ;
$$

(ii) $\rho$ is "linear", i.e., $\rho$ can be extended to a linear map (also denoted by $\rho$ ) from $U$ into $U^{\prime}$.

Let $\mathfrak{g}$ and $\mathfrak{g}^{\prime}$ be the Lie algebra of $G$ and $G^{\prime}$, respectively. Take $\omega_{0} \in$ $\Omega$ and put $\omega_{0}^{\prime}=\rho\left(\omega_{0}\right)$. We denote by $\mathfrak{f}^{\prime}=\mathfrak{f}_{\omega_{0}}\left(\right.$ resp. $\left.\mathfrak{f}^{\prime}=\mathfrak{f}_{\omega_{0}}^{\prime}\right)$ the subalgebra 
of $g$ (resp. $\left.\mathfrak{g}^{\prime}\right)$ corresponding to the isotropy subgroup $K=K_{\omega_{0}}$ (resp. $K^{\prime}=$ $K^{\prime}{ }_{\omega^{\prime} 0}$ ) of $G$ (resp. $G^{\prime}$ ) at $\omega_{0}$ (resp. $\left.\omega_{0}^{\prime}\right)$. Then, the condition (i) implies that one has a monomorphism of $g$ into $g^{\prime}$ (denoted again by $\rho$ ) such that

$$
\rho\left(\mathfrak{k}_{\omega_{0}}\right) \subset \mathfrak{H}_{\omega / 0^{\prime}}^{\prime} .
$$

Conversely, if one has a monomorphism $\rho: \mathfrak{g} \rightarrow \mathfrak{g}^{\prime}$ satisfying $\left(C_{0}\right)$ (for given $\left.\omega_{0} \in \Omega, \omega_{0}^{\prime} \in \Omega^{\prime}\right)$, then, using the corresponding local monomorphism $\rho$ : $G \rightarrow G^{\prime}$, one can define an equivariant injection $\rho: \Omega \rightarrow \Omega^{\prime}$ by putting $\rho\left(g\left(\omega_{0}\right)\right)$ $=\rho(g)\left(\omega_{0}^{\prime}\right)$. (Note that such a monomorphism $\rho: g \rightarrow g^{\prime}$ is not uniquely determined by the corresponding injection $\rho: \Omega \rightarrow \Omega^{\prime}$.)

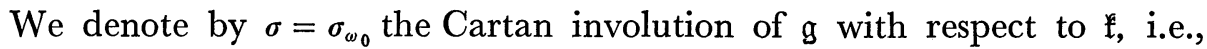
the (unique) involution of $\mathfrak{g}$ such that $\mathfrak{f}=\{X \in \mathfrak{g} \mid \sigma(X)=X\}$. Similarly, let $\sigma^{\prime}=\sigma_{\omega / 0}^{\prime}$ be the Cartan involution of $g^{\prime}$ with respect to $\mathfrak{f}^{\prime}$. Then clearly the condition $\left(C_{0}\right)$ is implied by the condition

$$
\rho \circ \sigma_{\omega_{0}}=\sigma_{\omega \prime 0}^{\prime} \circ \rho .
$$

It is well-known (see, e.g., [3a]) that, in general, an equivariant injection $\rho: \Omega \rightarrow \Omega^{\prime}$ of symmetric spaces comes from a monomorphism of $\mathrm{g}$ satisfying $(C)$ if and only if it is an isometry and $\rho(\Omega)$ is totally geodesic in $\Omega^{\prime}$ (for a suitable choice of the invariant Riemannian metrics on $\Omega$ and $\Omega^{\prime}$ ). It should also be noted that, in our case, if one denotes by $t$ the adjoint with respect to an inner product $\langle>$ on $U$ for which the condition (1) makes sense, then one has ${ }^{t} G=G,{ }^{t} \mathrm{~g}=\mathrm{g}$, and the map $X \longmapsto-{ }^{t} X(X \in \mathrm{g})$ is a Cartan involution of $\mathrm{g}$. It follows that a linear imbedding $\rho: \Omega \rightarrow \Omega^{\prime}$ comes from a monomorphism of $\mathrm{g}$ satisfying $(C)$ if and only if one can find a $K$ - (resp. $K^{\prime}-$ ) invariant inner product \langle\rangle (resp. \langle\rangle$^{\prime}$ ) on $U$ (resp. $U^{\prime}$ ) satisfying (1) for $\Omega$ (resp. $\Omega^{\prime}$ ) and such that

$$
\langle\rho(x), \rho(y)\rangle^{\prime}=\langle x, y\rangle \quad \text { for all } x, y \in U .
$$

2.2. In the following, we fix $\omega_{0} \in \Omega$ (and hence $\mathfrak{t}=\mathfrak{f}_{\omega_{0}}, \sigma=\sigma_{\omega_{0}}$ ) once and for all. Our problem is then to find all pairs $\left(\rho, \omega_{0}^{\prime}\right)$ formed of a monomorphism $\rho: \mathfrak{g} \rightarrow \mathfrak{g}^{\prime}$ and $\omega_{0}^{\prime} \in \Omega^{\prime}$ satisfying the condition $\left(C_{0}\right)$ and giving rise to a linear imbedding $\rho: \Omega \rightarrow \Omega^{\prime}$; a solution $\left(\rho, \omega_{0}^{\prime}\right)$ satisfying the stronger condition $(C)$ will be called a strong solution. As we mentioned in the Introduction, we shall consider this problem only for the case where $\Omega^{\prime}$ is of classical type, but in a slightly extended form, by dropping the injectivity of $\rho$ and the effectiveness of $G$ and $G^{\prime}$. Since the case $\mathscr{K}=\boldsymbol{R}$ or $\boldsymbol{K}$ can 
be deduced from the case $\mathscr{K}=\boldsymbol{C}$ (see 6 ), we shall henceforth restrict ourselves to the case $\Omega^{\prime}=\mathscr{P}(n, C)$. Thus, changing the notation, we let $G$ be a connected reductive Lie group acting on $U$ through a representation $\pi: G \rightarrow$ $G L(U)$, such that $\pi(G)=\operatorname{Aut}_{0}(\Omega), \pi^{-1}(1)=K_{0}$ : compact, and let $K$ be the isotropy subgroup of $G$ at $\omega_{0}$, which is again a maximal compact subgroup of $G$. On the other hand, let $V$ be a complex vector-space of dimension $n$ and set $G^{\prime}=G L(V), \Omega^{\prime}=\mathscr{P}(V)$ (the cone of all positive-definite hermitian forms on $V$ ), $U^{\prime}=\mathscr{H}(V)$ (the space of all hermitian forms on $V$ ), the action of $g^{\prime} \in G^{\prime}$ on $U^{\prime}$ being defined by

$$
\left(g^{\prime}(F)\right)(x, y)=F\left(g^{\prime-1} x, g^{\prime-1} y\right) \quad(x, y \in V)
$$

for $F \in U^{\prime}$. Then, our problem can be formulated as follows:

Problem. For a given pair $\left(\mathfrak{g}, \omega_{0}\right)$, find all triples $(V, \rho, F)$ formed of a representation $(V, \rho)$ of the Lie algebra $g$ and $F \in \mathscr{P}(V)$, such that $\rho(X)$ is skewhermitian with respect to $F$ for all $X \in \mathbb{1}$ and that the induced equivariant map $\rho$ : $\Omega \rightarrow \Omega^{\prime}=\mathscr{P}(V)$ is linear.

A solution $(V, \rho, F)$ is called a strong solution, if it satisfies the stronger condition

$$
\rho(\sigma(X))=-\rho(X)^{*} \quad \text { for all } X \in \mathfrak{g},
$$

where $\sigma$ is the Cartan involution of $g$ with respect to $f$ and $*$ denotes the adjoint with respect to the hermitian form $F$. Two triples $(V, \rho, F)$ and $\left(V^{\prime}, \rho^{\prime}, F^{\prime}\right)$ are said to be equivalent (resp. conjugate or anti-equivalent), if there exists a linear (resp. antilinear) isomorphism $\phi: V \rightarrow V^{\prime}$ such that one has

$$
\left\{\begin{array}{l}
\rho^{\prime}(X)=\phi \circ \rho(X) \circ \phi^{-1} \quad \text { for all } X \in \mathfrak{g}, \\
F^{\prime}(x, y)=F\left(\phi^{-1}(x), \phi^{-1}(y)\right)\left(\text { resp. }=F\left(\phi^{-1}(y), \phi^{-1}(x)\right)\right) \\
\text { for all } x, y \in V .
\end{array}\right.
$$

It is clear that if $(V, \rho, F)$ and $\left(V^{\prime}, \rho^{\prime}, F^{\prime}\right)$ are equivalent or conjugate and if one of them is a (strong) solution, then so is also the other. Since $g$ is real, it is clear that for any solution $(V, \rho, F)$ one also has a solution conjugate to it. A solution conjugate to itself is called a self-conjugate solution. For two triples $\left(V_{i}, \rho_{i}, F_{i}\right)(i=1,2)$ with $\rho_{i}: \mathfrak{g} \rightarrow \mathfrak{g l}\left(V_{i}\right), \quad F_{i} \in \mathscr{P}\left(V_{i}\right)$, the triple $\left(V_{1} \oplus V_{2}, \rho_{1} \oplus \rho_{2}, F_{1} \oplus F_{2}\right)$ is called the direct sum of them. The direct sum of $\left(V_{i}, \rho_{i}, F_{i}\right)(i=1,2)$ is a (strong) solution if and only if both of them are (strong) solutions. A triple $(V, \rho, F)$ is called irreducible (resp. 
primary) if the representation $\rho$ of $g$ is irreducible (resp. primary, i.e., decomposable into a direct sum of mutually equivalent irreducible representations).

2.3. Now, to state our main results, we introduce some more notation. From the decomposition (2) of $\Omega$ one obtains the following decomposition of the Lie algebra $g$ of $G$ :

$$
\mathfrak{g}=\mathfrak{l}_{0} \oplus \mathfrak{g}_{1} \oplus \cdots \oplus \mathfrak{g}_{\ell},
$$

where $\mathfrak{f}_{0}$ is the Lie algebra of the maximal compact normal subgroup $K_{0}$ of $G$ and $g_{i}$ is the Lie algebra of $G_{i}=\operatorname{Aut}_{0}\left(\Omega_{i}\right)(1 \leqslant i \leqslant t)$. We denote the semisimple (resp. abelian) part of $\mathfrak{g}_{i}$ by $\mathfrak{g}_{i}^{S}$ (resp. $\mathfrak{a}_{0 i}$ ) and put

$$
\mathfrak{g}^{S}=\sum_{i=1}^{t} \mathfrak{g}_{i}^{S}, \quad \mathfrak{a}_{0}=\sum_{i=1}^{t} \mathfrak{a}_{0 i}
$$

Then one has $\mathfrak{g}=\mathfrak{f}_{0} \oplus \mathfrak{a}_{0} \oplus \mathfrak{g}^{S}$. One writes also

$$
\omega_{0}=\sum_{i=1}^{t} \omega_{0 i}, \quad \omega_{0 i} \in \Omega_{i} .
$$

Theorem 1. (a) A solution $(V, \rho, F)$ of the above problem can be decomposed uniquely into the direct sum of primary solutions $\left(V^{(i)}, \rho^{(i)}, F^{(i)}\right)$, corresponding to the primary components of the representation $\rho$. The solution $(V, \rho, F)$ is a strong solution if and only if all its primary components $\left(V^{(i)}, \rho^{(i)}, F^{(i)}\right)$ are strong solutions.

(b) A primary solution $(V, \rho, F)$ can be expressed in the following form

$$
\left\{\begin{array}{l}
V=V_{1} \otimes V_{0}, \\
\rho=\rho_{1} \otimes 1+1 \otimes \rho_{0}, \\
F=F_{1} \otimes F_{0},
\end{array}\right.
$$

where $\left(V_{1}, \rho_{1}, F_{1}\right)$ is an irreducible strong solution for $\left(\mathfrak{g}_{i_{0}}, \omega_{0 i_{0}}\right)$ for some index $i_{0}$, $1 \leqslant i_{0} \leqslant t, V_{0}$ is a (finite-dimensional) complex vector-space, $F_{0} \in \mathscr{P}\left(V_{0}\right)$, and $\rho_{0}$ is a primary representation of $\mathfrak{\mathfrak { t }}_{0} \oplus \mathfrak{a}_{0}$ into $\mathfrak{u}\left(V_{0}, F_{0}\right)$ (the Lie algebra of all skewhermitian transformations of $V_{0}$ with respect to $F_{0}$ ). Here we understand that the representations $\rho_{1}$ and $\rho_{0}$ are extended in a trivial manner to the representations of $\mathrm{g}$, which are denoted by the same letters ${ }^{2}$. Conversely, any triple $(V, \rho, F)$ defined

2) Let $g_{0}$ be an ideal of a Lie algebra $\mathfrak{g}$ such that there exists a unique complementary ideal $\mathfrak{g}_{0}^{\prime}$ such that $\mathfrak{g}=\mathfrak{g}_{0} \oplus \mathfrak{g}_{0}^{\prime}$. Then, any representation of $\rho_{0}$ of $\mathfrak{g}_{0}$ can be extended (in a unique manner) to a representation of $g$ by putting $\rho_{0}(X)=0$ for $X \in \mathfrak{g}_{0}^{\prime}$. The representation of $\mathfrak{g}$ thus obtained is called a "trivial extension" of $\rho_{0}$ and is usually denoted by the same letter $\rho_{0}$. 
in this manner is a primary solution for $\left(\mathrm{g}, \omega_{0}\right)$. The primary solution $(V, \rho, F)$ is a strong solution if and only if one has $\rho_{0}\left(\mathfrak{a}_{0}\right)=\{0\}$.

It follows that any solution $(V, \rho, F)$ is decomposable into a direct sum of irreducible solutions (though such a decomposition is not unique). This fact can trivially be verified for a strong solution, for in that case the condition $\left(C^{\prime}\right)$ implies that, if $W$ is any $\rho(\mathfrak{g})$-invariant subspace of $V$, then the orthogonal complement $W^{\perp}$ of $W$ in $V$ (with respect to $F$ ) is also $\rho(\mathfrak{g}$ )invariant. The proof of Theorem 1 for the general case will be given in 3-5.

Theorem 1 also implies that, if $(V, \rho, F)$ is a solution, then $\rho$ is a "commutative sum" of two representations $\rho^{1}$ and $\rho^{03}$, where $\left(V, \rho^{1}, F\right)$ is a strong solution with $\rho^{1}\left(\mathfrak{F}_{0}\right)=0$ and $\rho^{0}$ is a trivial extension of a representation of $\mathfrak{f}_{0} \oplus \mathfrak{a}_{0}$ into $\mathfrak{t}(V, F)$. Thus the representations $\rho$ and $\rho^{1}$ induce the same map of $\Omega$ into $\Omega^{\prime}=\mathscr{P}(V)$, so that the image $\rho(\Omega)$ is always totally geodesic in $\Omega^{\prime}$. It should also be noted that, when the representation $\rho$ comes from an $\boldsymbol{R}$-rational homomorphism of an algebraic group defined over $\boldsymbol{R}$, the condition $\rho^{0}\left(\mathfrak{a}_{0}\right)=\{0\}$ is automatically satisfied, so that $(V, \rho, F)$ is a strong solution.

A key step in the proof of Theorem 1 is to show that for an irreducible solution $\left(V_{1}, \rho_{1}, F_{1}\right)$ the hermitian form $F_{1}$ is uniquely determined up to a positive scalar multiple by the representation $\left(V_{1}, \rho_{1}\right)$. This fact, which is again trivial for strong solutions, will be proved in 5 by using the classification given in 4 . This uniqueness, along with Theorem 1, will imply the following

Theorem 2. The equivalence class of a solution $(V, \rho, F)$ is uniquely determined only by the equivalence class of the representation $\rho$.

\section{Reduction of the problem.}

3.1. Our first step is to reduce the problem according to the decomposition (8) of the abelian part $a_{0}$. The notation being as in 2.3 , let $Z_{i}$ be the unique generator of $\mathfrak{a}_{0 i}$ such that $\exp \left(\lambda Z_{i}\right)\left(\in G_{i}\right)$ is the dilatation $x \longmapsto$ $e^{\lambda} x$ in $U_{i}(1 \leqslant i \leqslant t)$. (In other words, $Z_{i}$ viewed as a linear transformation

3) If $\rho^{\prime}$ and $\rho^{\prime \prime}$ are two representations of a Lie algebra $g$ on the same representationspace $V$ such that $\rho^{\prime}(X)$ and $\rho^{\prime \prime}\left(X^{\prime}\right)$ commute with each other for all $X, X^{\prime} \in \mathfrak{g}$, then the sum $\rho=\rho^{\prime}+\rho^{\prime \prime}$ is also a representation of $\mathfrak{g}$ on $V$, which we call a "commutative sum" of $\rho^{\prime}$ and $\rho^{\prime \prime}$. 
of $U_{i}$ is the identity transformation $1_{U_{i}}$ ) Then one has

$$
\exp \left(\sum_{i} \lambda_{i} Z_{i}\right)\left(\sum_{i} x_{i}\right)=\sum_{i} e^{\lambda_{i}} x_{i}
$$

for all $\lambda_{i} \in \boldsymbol{R}, \quad x_{i} \in U_{i}, 1 \leqslant i \leqslant t$. Hence, if $(V, \rho, F)$ is a solution for $\left(\mathfrak{g}, \omega_{0}\right)$, one has from the conditions (i), (ii)

$$
\begin{aligned}
\exp \left(\sum_{i} \lambda_{i} \rho\left(Z_{i}\right)\right)(F) & =\rho\left(\exp \left(\sum_{i} \lambda_{i} Z_{i}\right)\left(\omega_{0}\right)\right) \\
& =\rho\left(\sum_{i} e^{\lambda_{i}} \omega_{0 i}\right) \\
& =\sum_{i} e^{\lambda_{i}} \rho\left(\omega_{0 i}\right) .
\end{aligned}
$$

Hence, putting $Z_{i}^{\prime}=\rho\left(Z_{i}\right), F_{i}=\rho\left(\omega_{0 i}\right)$, one has

$$
F\left(\exp \left(-\sum_{i} \lambda_{i} Z_{i}^{\prime}\right) x, \exp \left(-\sum_{i} \lambda_{i} Z_{i}^{\prime}\right) y\right)=\sum_{i} e^{\lambda_{i}} F_{i}(x, y)
$$

for all $x, y \in V$. Differentiating both sides of this equality with respect to the variable $\lambda_{i}$, one obtains

$$
-F\left(Z_{i}^{\prime} x^{\prime}, y^{\prime}\right)-F\left(x^{\prime}, Z_{i}^{\prime} y^{\prime}\right)=F_{i}(x, y),
$$

where $x^{\prime}=\exp \left(\sum_{j \neq i} \lambda_{j} Z_{j}^{\prime}\right) x, y^{\prime}=\exp \left(\sum_{j \neq i} \lambda_{j} Z_{j}^{\prime}\right) y$. From this, one obtains immediately the following relations:

$$
\begin{gathered}
F\left(Z_{i}^{\prime} x, y\right)+F\left(x, Z_{i}^{\prime} y\right)=-F_{i}(x, y), \\
F_{i}\left(Z_{j}^{\prime} x, y\right)+F_{i}\left(x, Z_{j}^{\prime} y\right)=-\delta_{i j} F_{i}(x, y)
\end{gathered}
$$

for all $x, y \in V$ and $1 \leqslant i, j \leqslant t$.

Now, since $F_{i}$ belongs to the closure of $\rho(\Omega)$, the hermitian form $F_{i}$ is positive semi-definite. Hence

$$
W_{i}^{\prime}=\left\{x \in V \mid F_{i}(x)=0\right\}
$$

is a linear subspace of $V$. Let $W_{i}$ be the orthogonal complement of $W_{i}^{\prime}$ with respect to $F$. Then one has

$$
\left\{\begin{array}{l}
V=W_{i} \oplus W_{i}^{\prime}, \\
F_{i}\left|W_{i} \gg 0, F_{i}\right| W_{i}^{\prime}=0 .
\end{array}\right.
$$

From (12) and (13) it is clear that both $W_{i}$ and $W_{i}^{\prime}$ are invariant under $Z_{i}^{\prime}$. Moreover, from (13) one sees that $Z_{i}^{\prime} \mid W_{i}+\frac{1}{2} 1_{W_{i}}$ is skew-hermitian with respect to $F_{i} \mid W_{i}$. Hence $Z_{i}^{\prime} \mid W_{i}$ is diagonalizable and all its eigen-values have 
the real part $=-\frac{1}{2}$. Similarly, from (12) one sees that $Z_{i}^{\prime} \mid W_{i}^{\prime}$ is skewhermitian with respect to $F \mid W_{i}^{\prime}$, hence also diagonalizable, and all its eigenvalues are purely imaginary. Since $Z_{1}^{\prime}, \cdots, Z_{t}^{\prime}$ are mutually commutative, it follows that the decomposition (14) is invariant under all $Z_{j}^{\prime}, 1 \leqslant j \leqslant t$.

We now assert that, for $i \neq j, W_{i}$ and $W_{j}$ are orthogonal with respect to $F$. In fact, since one has $\left(F_{i}+F_{j}\right) \mid\left(W_{i}+W_{j}\right) \gg 0$, one sees from (13) by the same argument as above that all eigen-values of $\left(Z_{i}^{\prime}+Z_{j}^{\prime}\right) \mid\left(W_{i}+W_{j}\right)$ have the real part $=-\frac{1}{2}$. Since one has

$$
W_{i}+W_{j}=W_{i} \cap W_{j}+W_{i} \cap W_{j}^{\prime}+W_{j} \cap W_{i}^{\prime},
$$

this implies that $W_{i} \cap W_{j}=\{0\}$, and so $W_{i} \subset W_{j}^{\prime}, W_{j} \subset W_{i}^{\prime}$, which proves our assertion.

On the other hand, since $F=\sum F_{i}$ is positive-definite, it is clear that $\bigcap_{i=1}^{t} W_{i}^{\prime}=\{0\}$. Hence, one obtains the following orthogonal decomposition

$$
V=W_{1} \oplus \cdots \oplus W_{t}
$$

If $P_{i}$ denotes the orthogonal projection of $V$ onto $W_{i}$, then $Z_{i}^{\prime}+\frac{1}{2} P_{i}$ is skew-hermitian (with respect to $F$ ) and one has

$$
F_{i}(x, y)=F\left(P_{i} x, P_{i} y\right) \quad \text { for } x, y \in V .
$$

3. 2. Now, since $\mathfrak{a}_{0}$ is in the center of $\mathfrak{g}$, it is clear that the decomposition (15) is invariant under $\rho(\mathrm{g})$. Hence the solution $(V, \rho, F)$ decomposes into the direct sum of the solutions corresponding to $W_{i}, 1 \leqslant i \leqslant t$. Thus the whole problem can be reduced to the case $V=W_{i_{0}}$ for some $i_{0}$. For brevity when this condition is satisfied, i.e., when one has $\rho\left(\mathfrak{a}_{0 i}\right) \subset \mathfrak{t}(V, F)$ for all $i \neq i_{0}$, we shall say that the solution $(V, \rho, F)$ belongs to the component $g_{i_{0}}$.

Lemma. Let $(V, \rho, F)$ be a solution such that one has $\rho\left(\mathfrak{a}_{0 i}\right) \subset \mathfrak{H}(V, F)$. Then, one has $\rho\left(\mathfrak{g}_{i}^{S}\right)=\{0\}$ and $\rho\left(U_{i}\right)=\{0\}$.

Proof. Let $g \in G_{i}$. Then, since $\rho\left(\omega_{0 i}\right)=0$, one has

$$
\begin{aligned}
\rho(g)(F)=\rho\left(g\left(\omega_{0}\right)\right) & =\rho\left(g\left(\omega_{0 i}\right)+\sum_{j \neq i} \omega_{0 j}\right) \\
& =\rho\left(g\left(\omega_{0 i}\right)\right)+F .
\end{aligned}
$$

Writing $g$ in the form $g=g^{S} \exp \left(\lambda Z_{i}\right)$ with $g^{S} \in G_{i}^{S}, \lambda \in \boldsymbol{R}$, one has 


$$
\rho(g)(F)=\rho\left(g^{S}\right)(F)=e^{\lambda} \cdot \rho\left(g^{S}\left(\omega_{0 i}\right)\right)+F
$$

It follows that

$$
\rho\left(g^{S}\right)(F)=F, \quad \rho\left(g^{S}\left(\omega_{0 i}\right)\right)=0
$$

for all $g^{S} \in G_{i}^{S}$. The first relation implies that $\rho\left(G_{i}^{S}\right) \subset U(V, F)$. But, since $G_{i}^{S}$ is either a non-compact simple Lie group or $=\{1\}$, one concludes that $\rho\left(\mathfrak{g}_{i}^{S}\right)=\{0\}$. On the other hand, since $g^{S}\left(\omega_{0 i}\right)\left(g^{S} \in G_{i}^{S}\right)$ generate linearly the whole space $U_{i}$, the second relation implies that $\rho\left(U_{i}\right)=\{0\}$, q.e.d.

It follows that, if $(V, \rho, F)$ is a solution belonging to the component $\mathfrak{g}_{i_{0}}$, then one has $\rho\left(\mathfrak{g}_{i}^{S}\right)=\{0\}$ and $\rho\left(U_{i}\right)=\{0\}$ for all $i \neq i_{0}$, so that $\left(V, \rho \mid g_{i_{0}}, F\right)$ is clearly a solution for $\left(\mathfrak{g}_{i_{0}}, \omega_{0 i_{0}}\right)$ and $\rho$ is a commutative sum of two representations $\rho^{1}$ and $\rho^{0}$ which are the trivial extensions of $\rho / g_{i_{0}}$ and $\rho \mid\left(\mathfrak{f}_{0} \oplus \sum_{i \neq i_{0}} \mathfrak{a}_{0 i}\right)$, respectively.

Our result can be summarized as follows.

Proposition 1. A solution for $\left(\mathfrak{g}, \omega_{0}\right)$ can be decomposed uniquely into the direct sum of solutions belonging to one of the components $\mathrm{g}_{i}(1 \leqslant i \leqslant t)$. If $(V, \rho, F)$ is a solution belonging to a component $\mathfrak{g}_{i}$, then $\rho$ can be expressed as a commutative sum of two representations $\rho^{1}$ and $\rho^{0}$, where $\rho^{1}$ is a trivial extension ${ }^{2}$ of a representation of $\mathfrak{g}_{i}$ such that $\left(V, \rho^{1} \mid \mathfrak{g}_{i}, F\right)$ is a solution for $\left(\mathfrak{g}_{i}, \omega_{0 i}\right)$ and $\rho^{0}$ is a trivial extension of a representation of $\mathfrak{\mathfrak { t }}_{0} \oplus \mathfrak{a}_{0}$ into $\mathfrak{u}(V, F)$.

The decomposition $\rho=\rho^{1}+\rho^{0}$ mentioned in Proposition 1 is not unique, but will become unique if $\rho^{1}$ is normalized by the condition $\rho^{1}\left(Z_{i}\right)=$ $-\frac{1}{2} 1_{V}$.

3. 3. By virtue of Proposition 1, the proofs of Theorems 1 and 2 can be reduced to the case where $\Omega$ is irreducible and $\mathrm{g}$ is the Lie algebra of $\operatorname{Aut}_{0}(\Omega)$, and where the representation $\rho$ is "normalized" by the condition

$$
\rho\left(1_{U}\right)=-\frac{1}{2} 1_{V}
$$

Actually, we shall prove in 5 the following

Proposition 2. Suppose $\Omega$ is irreducible and $\mathrm{g}$ is the Lie algebra of $\operatorname{Aut}_{0}(\Omega)$. Let $(V, \rho, F)$ be a solution for $\left(\mathrm{g}, \omega_{0}\right)$ normalized by the condition (17). Then:

(a) If $V=\Sigma V^{(i)}$ is the decomposition of $V$ into the direct sum of primary 
components with respect to the representation $\rho$, then the $V^{(i)}$ 's are mutually orthogonal with respect to $F$.

(b) $A$ normalized primary solution $(V, \rho, F)$ can be expressed in the form

$$
\left\{\begin{array}{l}
V=V_{1} \otimes V_{0}, \\
\rho=\rho_{1} \otimes 1 \\
F=F_{1} \otimes F_{0}
\end{array}\right.
$$

where $\left(V_{1}, \rho_{1}, F_{1}\right)$ is an irreducible strong solution, $V_{0}$ is a complex vector-space and $F_{0} \in \mathscr{P}\left(V_{0}\right)$.

(c) For an irreducible strong solution $\left(V_{1}, \rho_{1}, F_{1}\right), F_{1}$ is uniquely determined by $\left(V_{1}, \rho_{1}\right)$ up to a positive scalar multiple.

It is immediate that Theorem 1 follows from Propositions 1 and 2, (a), (b). Also, assuming Proposition 2, Theorem 2 can be proved as follows. Let $(V, \rho, F)$ and $\left(V^{\prime}, \rho^{\prime}, F^{\prime}\right)$ be two solutions for $\left(\mathfrak{g}, \omega_{0}\right)$ and suppose that $\rho$ and $\rho^{\prime}$ are equivalent. By Theorem 1, (a) we may assume that both $\rho$ and $\rho^{\prime}$ are primary. Then, by Theorem 1, (b) we may write

$$
\left\{\begin{array} { l } 
{ V = V _ { 1 } \otimes V _ { 0 } , } \\
{ \rho = \rho _ { 1 } \otimes \rho _ { 0 } , } \\
{ F = F _ { 1 } \otimes F _ { 0 } , }
\end{array} \quad \left\{\begin{array}{l}
V^{\prime}=V_{1}^{\prime} \otimes V_{0}^{\prime}, \\
\rho^{\prime}=\rho_{1}^{\prime} \otimes \rho_{0}^{\prime}, \\
F^{\prime}=F_{1}^{\prime} \otimes F_{0}^{\prime} .
\end{array}\right.\right.
$$

Let $\phi$ be a linear isomorphism of $V$ onto $V^{\prime}$ such that $\phi \circ \rho(X)=\rho^{\prime}(X) \circ \phi$ for all $X \in \mathrm{g}$. Since $\rho_{1}$ and $\rho_{1}^{\prime}$ are the trivial extensions of mutually equivalent irreducible representations of $g_{i_{0}}$, one has by Shur's lemma that $\phi=$ $\phi_{1} \otimes \phi_{0}$ with $\phi_{1}: V_{1} \rightarrow V_{1}^{\prime}, \phi_{0}: V_{0} \rightarrow V_{0}^{\prime}$ (cf. [3a], p. 441). Then, by Proposition 2 , (c), one has

$$
F_{1}^{\prime}\left(\phi_{1}(x), \phi_{1}(y)\right)=c F_{1}(x, y) \quad \text { for } x, y \in V_{1},
$$

with $c>0$. On the other hand, one can write

$$
F_{0}^{\prime}\left(\phi_{0}(x), \phi_{0}(y)\right)=F_{0}(x, T y) \quad \text { for } x, y \in V_{0}
$$

with a linear transformation $T$ of $V_{0}$, which is hermitian and positive-definite with respect to $F_{0}$ and commutes with $\rho_{0}(X)\left(X \in \mathfrak{f}_{0} \oplus \mathfrak{a}_{0}\right)$. Hence we may replace $\phi$ by $c^{-1 / 2} \phi_{1} \otimes\left(\phi_{0} \circ T^{-1 / 2}\right)$ to obtain the relation

$$
F^{\prime}(\phi(x), \phi(y))=F(x, y) \quad \text { for } x, y \in V .
$$

Therefore, $(V, \rho, F)$ and $\left(V^{\prime}, \rho^{\prime}, F^{\prime}\right)$ are equivalent. 
3.4. Under the assumption of Proposition 2, one has $\mathfrak{g}=\mathfrak{a}_{0}+\mathfrak{g}^{S}, \mathfrak{a}_{0}=$ $\boldsymbol{R} 1_{U}, \mathfrak{g}^{S}$ : simple. If $\mathfrak{g}^{S}=\{0\}$, i.e., $\Omega=\mathscr{P}(1, \boldsymbol{R})$, then Proposition 2 is trivially true. Hence, in the following, we shall assume that $g^{S} \neq\{0\}$. Then, since $\rho$ is normalized; $\rho$ is uniquely determined by its restriction $\rho^{S}$ on $g^{S}$.

Let $\mathfrak{g}^{S}=\mathfrak{i}+\mathfrak{m}^{S}$ be the Cartan decomposition of $\mathrm{g}^{S}$ corresponding to $\omega_{0}$ and let $a^{S}$ be a maximal abelian subalgebra of $\mathfrak{g}^{S}$ contained in $\mathfrak{m}^{S}$. Then $\mathfrak{a}=\mathfrak{a}_{0}+\mathfrak{a}^{S}$ is a maximal abelian subalgebra of $\mathfrak{g}$ contained in $\mathfrak{m}=\mathfrak{a}_{0}+\mathfrak{m}^{S}$. Let $(V, \rho, F)$ be a normalized solution for $\left(g, \omega_{0}\right)$ and put $\rho^{s}=\rho \mid g^{s}$. Then, as is well-known, one can find a basis $\left(v_{1}, \cdots, v_{n}\right)$ of $V$ over $\boldsymbol{C}$ such that

$$
\rho(X) v_{i}=\lambda_{i}(X) v_{i} \quad \text { for all } X \in \mathfrak{a}^{s},
$$

where $\lambda_{i}$ 's are real-valued linear forms on $\mathfrak{a}^{S}$, called the "restricted weights" of $\rho^{s}$. Similarly, one can find a basis $\left(u_{1}, \cdots, u_{m}\right)$ of $U$ over $\boldsymbol{R}$ such that

$$
\pi(X) u_{i}=\mu_{i}(X) u_{i} \quad \text { for all } X \in \mathfrak{a}^{s}
$$

with (real-valued) linear forms $\mu_{i}$ on $\mathfrak{a}^{S}$. Put $\omega_{0}=\sum c_{i} u_{i}$. Then, from the linearity of $\rho$ one has

$$
\rho\left(\exp (X) \omega_{0}\right)=\rho\left(\sum e^{\mu_{i}(X)} c_{i} u_{i}\right)=\sum e^{\mu_{i}(X)} c_{i} \rho\left(u_{i}\right) .
$$

On the other hand, if one puts $F\left(v_{i}, v_{j}\right)=a_{i j}$, the matrix of the hermitian form $\rho\left(\exp (X) \omega_{0}\right)$ in the basis $\left(v_{i}\right)$ is

$$
\operatorname{diag}\left(e^{-\lambda_{i}(X)}\right) \cdot\left(a_{i j}\right) \cdot \operatorname{diag}\left(e^{-\lambda_{i}(X)}\right)=\left(e^{-\left(\lambda_{i}(X)+\lambda_{j}(X)\right)} a_{i j}\right) .
$$

Since $a_{i i}>0$, this implies that $e^{-2 \lambda_{i}(X)}$ is a linear combination of the $e^{\mu_{j}(X)}$ 's $(1 \leqslant j \leqslant m)$. It follows that, for each $i, 1 \leqslant i \leqslant n$, there exists $a j, 1 \leqslant j \leqslant m$, such that one has $\lambda_{i}(X)=-\frac{1}{2} \mu_{j}(X)\left(X \in \mathfrak{a}^{S}\right)$. In general, when a representation $\rho^{S}$ of $\mathrm{g}^{S}$ has this property, we say that $\rho^{S}$ satisfies the condition $(L)$. It is clear that, if a representation $\rho^{s}$ satisfies the condition $(L)$, then so does also every irreducible component of $\rho^{s}$. In the next section, we shall determine case-by-case all irreducible representations $\rho^{s}$ of $g^{S}$ satisfying the condition $(L)$, and show that for each such irreducible representation $\rho^{s}$ one can find at least one $F \in \mathscr{P}(V)$ such that $(V, \rho, F)$ is a strong solution for $\left(\mathfrak{g}, \omega_{0}\right)$, where $\rho$ is the unique extension of $\rho^{s}$ to $g$ normalized by (17). The uniqueness of such $F$ will be shown later in 5 . 


\section{Determination of irreducible representations $\rho^{S}$ satisfying} the condition $(L)$.

In this section, we use the following notation. $h$ is a Cartan subalgebra of $\mathrm{g}^{S}$ invariant under $\sigma$ and containing $\mathfrak{a}^{S}$. Let $\mathfrak{g}_{C}^{S}=\mathfrak{g}^{S} \otimes_{\boldsymbol{R}} \boldsymbol{C}$ be the complexification of $\mathfrak{g}^{S}$ and put $\mathfrak{h} \boldsymbol{c}=\boldsymbol{C} \mathfrak{h}$. Except for the case $2^{\circ}$ (were $\mathfrak{g}_{\boldsymbol{C}}^{S}$ is not simple), let $\left(\alpha_{1}, \cdots, \alpha_{l}\right)(l=\operatorname{dim} \mathfrak{h})$ be a $\sigma$-fundamental system of roots with respect to $\mathfrak{h} c^{4)}$ and put

$$
\mathfrak{h}^{0}=\left\{X \in \mathfrak{h} \boldsymbol{C} \mid \alpha_{i}(X) \in \boldsymbol{R} \text { for } 1 \leqslant i \leqslant l\right\} .
$$

Let $\left(\tilde{\omega}_{0}, \cdots, \widetilde{\omega}_{l}\right)$ be the corresponding system of fundamental weights, and put $\eta_{i}=\widetilde{\omega}_{i} \mid \mathfrak{a}^{S}$. Let $\left(V, \rho^{S}\right)$ be an irreducible representation of $\mathrm{g}^{S}$ satisfying the condition $(L)$ and $\rho$ the unique normalized irreducible representation of $g$ extending $\rho^{s}$. We denote by $\Lambda_{\rho} s$ the highest weight of $\rho^{s}$ and put $\lambda_{\rho} s=$ $\Lambda_{\boldsymbol{\rho}} s \mid \mathfrak{a}^{s}$.

In the classical cases $1^{\circ}-3^{\circ}$, one has

$$
\Omega=\mathscr{P}(\nu, \mathscr{K}), \mathrm{g}^{S}=\mathfrak{g}((\nu, \mathscr{K})(\nu \geqslant 2) \text { with } \mathscr{K}=\boldsymbol{R}, \boldsymbol{C}, \boldsymbol{K},
$$

and one may take

$$
\mathfrak{a}^{S}=\left\{X=\operatorname{diag}\left(\xi_{1}, \cdots, \xi_{\nu}\right) \mid \xi_{i} \in \boldsymbol{R}, \sum_{i} \xi_{i}=\mathbf{0}\right\} .
$$

In each case, we denote by $\boldsymbol{V}$ a real vector-space of dimension $\nu \cdot \operatorname{dim} \mathscr{K}$, having a structure of a $g$ - $\mathscr{K}$-module, $g$ and $\mathscr{K}$ acting on $\boldsymbol{V}$ from the left and right, respectively. Then $\mathfrak{g}^{S}$ may be written as $\mathrm{g}^{S}=\mathfrak{g l}(\boldsymbol{V} / \mathscr{K})$.

Case $1^{\circ}$. One has $\mathfrak{h}^{0}=\mathfrak{a}^{S}, l=\nu-1$, and

$$
\eta_{i}=\xi_{1}+\cdots+\xi_{i} \quad \text { for } 1 \leqslant i \leqslant \nu-1,
$$

where $\xi_{i}$ stands for the linear form on $\mathfrak{a}^{s}$ assigning to a diagonal matrix $X \in \mathfrak{a}^{S}$ its $(i, i)$ component. Since the action of $G^{S}=S L(\nu, \boldsymbol{R})$ on $\Omega$ is given by $g(\omega)={ }^{t} g^{-1} \cdot \omega \cdot g^{-1}$, the restricted weights of the representation $\pi^{S}=\pi \mid g^{S}$ are of the form $\mu=-\left(\xi_{j}+\xi_{j^{\prime}}\right)$. Hence $-\frac{1}{2} \mu$ can be a restricted weight of $\rho^{s}$ only if $j=j^{\prime}$, and the condition $(L)$ implies that $\lambda_{\rho} s=\xi_{1}=\eta_{1}$, i.e., $\Lambda_{\rho} s=$ $\widetilde{\omega}_{1}$. This means that we may identify $V$ with $V_{C}$ in such a way that $\rho$ becomes the canonical injection $\mathfrak{g}=\mathfrak{g l}(\boldsymbol{V} / \boldsymbol{R}) \rightarrow \mathfrak{g l}(V / \boldsymbol{C})$, which we will simply denote by $i d$. Moreover, for this representation, any given $\omega_{0} \in \mathscr{P}(\boldsymbol{V} / \boldsymbol{R})$

4) For the notion of $\sigma$-fundamental system, see Annals of Math., Vol. 71 (1960), p. 80. 
can naturally be extended to an $F \in \mathscr{P}(V / C)$, and the triple $(V, i d ., F)$ is clearly a strong solution for $\left(\mathfrak{g}, \omega_{0}\right)$.

Case $2^{\circ}$. In this case, one has

$$
\boldsymbol{V} \boldsymbol{c}=V^{\prime} \oplus V^{\prime \prime}, V^{\prime \prime}=\bar{V}^{\prime},
$$

where $V^{\prime}$ and $V^{\prime \prime}$ are the $( \pm i)$-eigen-subspaces of the given complex structure on $\boldsymbol{V}$. Then one has $\mathfrak{g}_{\boldsymbol{C}}=\mathfrak{g}^{\prime} \oplus \mathfrak{g}^{\prime \prime}$ with $\mathfrak{g}^{\prime S}=\mathfrak{g l}\left(V^{\prime} / \boldsymbol{C}\right), \mathfrak{g}^{\prime \prime s}=\mathfrak{g l}\left(V^{\prime \prime} \mid \boldsymbol{C}\right)$, and, if one denotes by $p^{\prime}$ and $p^{\prime \prime}$ the projection of $g_{C}$ onto the factors $g^{\prime}$ and $\mathrm{g}^{\prime \prime}$, respectively, then the restriction of $p^{\prime}$ (resp. $p^{\prime \prime}$ ) on $g$ is a $C$-linear (resp. $\boldsymbol{C}$-antilinear) isomorphism of $\mathfrak{g}$ onto $\mathfrak{g}^{\prime}\left(\right.$ resp. $\left.\mathfrak{g}^{\prime \prime}\right)$. One has $\mathfrak{b} \boldsymbol{C}=\boldsymbol{C} p^{\prime}\left(\mathfrak{a}^{s}\right)$ $+C p^{\prime \prime}\left(\mathfrak{a}^{S}\right)$. The irreducible representation $\left(V, \rho^{S}\right)$ of $\mathrm{g}^{S}$ can be written in the form

$$
V=W^{\prime} \otimes W^{\prime \prime}, \quad \rho^{S}=\left(\rho^{\prime S} \circ p^{\prime}\right) \otimes\left(\rho^{\prime \prime S} \circ p^{\prime \prime}\right),
$$

where $\left(W^{\prime}, \rho^{\prime s}\right)$ and $\left(W^{\prime \prime}, \rho^{\prime \prime s}\right)$ are irreducible representations of $\mathrm{g}^{\prime s}$ and $\mathrm{g}^{\prime \prime s}$, respectively. If $\Lambda_{1}^{\prime}, \cdots, \Lambda_{n \prime}^{\prime}, n^{\prime}=\operatorname{dim} W^{\prime}\left(\operatorname{resp} . \Lambda_{1}^{\prime \prime}, \cdots, \Lambda_{n \prime \prime}^{\prime \prime}, n^{\prime \prime}=\operatorname{dim} W^{\prime \prime}\right)$ are the weights of $\rho^{\prime S}$ (resp. $\left.\rho^{\prime \prime S}\right)$ with respect to $p^{\prime}\left(\mathfrak{G}_{\boldsymbol{C}}\right)$ (resp. $p^{\prime \prime}\left(\mathfrak{h}_{\boldsymbol{C}}\right)$ ), then the weights of $\rho^{s}$ are given by $\Lambda_{i}^{\prime} \circ p^{\prime}+\Lambda_{i}^{\prime \prime} \circ p^{\prime \prime}$. By the similar argument, as in the case $1^{\circ}$, one sees from the condition $(L)$ that $\left(\Lambda_{i}^{\prime} \circ p^{\prime}+\Lambda_{i \prime}^{\prime \prime} \circ p^{\prime \prime}\right) \mid \mathfrak{a}^{S}=\xi_{j}$ for some $j$. On the other hand, one can choose the fundamental weights $\widetilde{\omega}_{i}^{\prime}$ (resp. $\left.\widetilde{\omega}_{i}^{\prime \prime}\right)$ of $\mathfrak{g}^{\prime S}$ (resp. $\left.\mathfrak{g}^{\prime \prime S}\right)$ with respect to $p^{\prime}\left(\mathfrak{h}_{\boldsymbol{c}}\right)$ (resp. $p^{\prime \prime}\left(\mathfrak{h}_{\boldsymbol{c}}\right)$ ) in such a way that $\eta_{i}=\widetilde{\omega}_{i}^{\prime} \circ p^{\prime}\left|\mathfrak{a}^{S}=\widetilde{\omega}_{i}^{\prime \prime} \circ p^{\prime \prime}\right| \mathfrak{a}^{S}$ are given by (21). It follows that one has either $\rho^{\prime S}=i d ., \rho^{\prime \prime} s=$ triv., or $\rho^{\prime S}=$ triv., $\rho^{\prime \prime s}=i d$.; in other words, $\left(V, \rho^{S}\right)$ can be identified with either $\left(V^{\prime}, p^{\prime} \mid g^{S}\right)$ or $\left(V^{\prime \prime}, p^{\prime \prime} \mid g^{S}\right)$. Again it is clear that $\omega_{0} \in \mathscr{P}(\boldsymbol{V} / \boldsymbol{C})$ determines an element $F \in \mathscr{P}\left(V^{\prime} / \boldsymbol{C}\right)$ in a natural manner and one gets two mutually conjugate strong solutions $\left(V^{\prime}, p^{\prime} \mid g, F\right)$ $\left(\sim\left(\boldsymbol{V} / \boldsymbol{C}, i d ., \omega_{0}\right)\right)$ and $\left(V^{\prime \prime}, p^{\prime \prime} \mid \mathrm{g}, \bar{F}\right)$.

Case $3^{\circ}$. As usual, we take a standard basis $(1, i, j, i j)$ of $\boldsymbol{K}$ over $\boldsymbol{R}$ and denote by $I, J$ the (real) linear transformations of $\boldsymbol{V}$ defined by the right multiplication of $i, j$, respectively. One has $l=2 \nu-1$ and one may take

$$
\mathfrak{h}=\left\{X+X^{\prime} I \mid X=\operatorname{diag}\left(\xi_{k}\right), X^{\prime}=\operatorname{diag}\left(\xi_{k}^{\prime}\right), \xi_{k}, \xi_{k}^{\prime} \in \boldsymbol{R}, \sum_{k} \xi_{k}=0\right\}
$$

Then a $\sigma$-fundamental system is given as follows:

$$
\left\{\begin{aligned}
\alpha_{2 k-1}\left(X+X^{\prime} I\right) & =2 \sqrt{-1} \xi_{k}^{\prime} & & (1 \leqslant k \leqslant \nu), \\
\alpha_{2 k}\left(X+X^{\prime} I\right) & =\left(\xi_{k}-\xi_{k+1}\right)-\sqrt{-1}\left(\xi_{k}^{\prime}+\xi_{k+1}^{\prime}\right) & & (1 \leqslant k \leqslant \nu-1) .
\end{aligned}\right.
$$


Hence one has

$$
\mathfrak{a}^{S}=\left\{X \in \mathfrak{h} \mid \alpha_{\imath}(X)=0 \text { for } i: \text { odd }\right\}
$$

and

$$
\left\{\begin{aligned}
\eta_{2 k-1} & =2\left(\xi_{1}+\cdots+\xi_{k-1}\right)+\xi_{k} & & (1 \leqslant k \leqslant \nu), \\
\eta_{2 k} & =2\left(\xi_{1}+\cdots+\xi_{k}\right) & & (1 \leqslant k \leqslant \nu-1) .
\end{aligned}\right.
$$

It follows again from condition $(L)$ that one has $\lambda_{\rho} s=\xi_{1}=\eta_{1}$, i.e., $\Lambda_{\rho} s=\widetilde{\omega}_{1}$. This means that $n=2 \nu$ and $V$ may be identified with $V$, viewed as a $2 \nu$ dimensional complex vector-space, and $\rho$ is given by the canonical injection $\mathfrak{g l}(\boldsymbol{V} / \boldsymbol{K}) \rightarrow \mathfrak{g l}(\boldsymbol{V} / \boldsymbol{C})$, denoted by $i d$. Moreover, for any $\boldsymbol{F} \in \mathscr{P}(\boldsymbol{V} / \boldsymbol{K})$, there corresponds uniquely an element $F \in \mathscr{P}(\boldsymbol{V} / \boldsymbol{C})$ such that one has

$$
\left\{\begin{aligned}
F(J x, J y) & =F(y, x) \\
\boldsymbol{F}(x, y) & =F(x, y)-F(x, J y) j \quad \text { for all } x, y \in \boldsymbol{V}
\end{aligned}\right.
$$

(see 6.1). At $\omega_{0}=\boldsymbol{F}$ the condition $\left(C^{\prime}\right)$ is trivially satisfied, and the above correspondence $\boldsymbol{F} \longmapsto F$ is clearly equivariant and linear. Thus, denoting by $F$ the element in $\mathscr{P}(\boldsymbol{V} / \boldsymbol{C})$ corresponding to $\omega_{0}=\boldsymbol{F}$, one obtains a strong solution $(\boldsymbol{V} / \boldsymbol{C}, i d ., F)$ for $\left(\mathfrak{g}, \omega_{0}\right)$.

Case $4^{\circ}$. Let $U$ be a real vector-space of dimension $m$ and $Q$ a quadratic form on $U$ of signature $(1, m-1) . \quad \Omega$ is given by one of the two connected components of $\{x \in U \mid Q(x)>0\}$, and one has $G^{S}=S O_{0}(U, Q)$. One has $\operatorname{dim} \mathfrak{h}=l=\left[\frac{m}{2}\right]$ and $\operatorname{dim} \mathfrak{a}^{S}=1$. If one enumerates the $\sigma$-fundamental system as shown in the diagram,

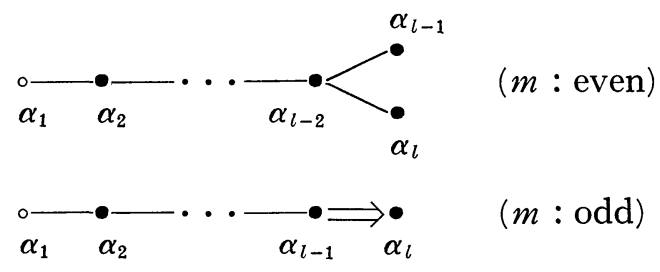

then one has

$$
\mathfrak{a}^{s}=\left\{X \in \mathfrak{h}^{0} \mid \alpha_{i}(X)=0 \text { for } 2 \leqslant i \leqslant l\right\} .
$$

The corresponding restricted fundamental weights are given as follows (cf. [1], Planche II, IV): 


$$
\eta_{i}= \begin{cases}\gamma_{1} & \text { for } 1 \leqslant i \leqslant l-2 \\ \frac{1}{2} \gamma_{1} & \text { for } i=l-1, l \quad(m: \text { even }),\end{cases}
$$

$$
\eta_{i}= \begin{cases}\gamma_{1} & \text { for } 1 \leqslant i \leqslant l-1 \\ \frac{1}{2} \gamma_{1} & \text { for } i=l \quad(m: \text { odd }) .\end{cases}
$$

Since the restricted weights of the (irreducible) representation $\pi^{S}$ of $\mathrm{g}^{S}$ are of the form $\mu=0, \pm \gamma_{1}$, one has from the condition $(L)$ that $\lambda_{\rho} s=\frac{1}{2} \gamma_{1}$, i.e., $\Lambda_{\rho} s=\widetilde{\omega}_{l-1}$ or $\widetilde{\omega}_{l}$ if $m$ is even, and $=\widetilde{\omega}_{l}$ if $m$ is odd. Hence $\left(V, \rho^{s}\right)$ is a spin representation.

To show that one can actually construct a strong solution $(V, \rho, F)$ for a spin representation $\rho^{s}$, let $C^{+}=C^{+}(U, Q)$ be the even part of the Clifford algebra, and let, be the canonical involution of $C^{+}$. Then the spin group $\widetilde{G}^{s}$ and the covering homomorphism $\phi: \widetilde{G}^{s} \rightarrow G^{s}$ are defined as follows:

$$
\begin{aligned}
& \widetilde{G}^{s}=\operatorname{Spin}(U, Q)=\left\{g \in C^{+} \mid g^{\prime} g=1, g U g^{-1}=U\right\}, \\
& \phi(g)(u)=g u g^{-1} \quad \text { for } u \in U, g \in \widetilde{G}^{s} .
\end{aligned}
$$

The Lie algebra $\mathrm{g}^{\mathcal{S}}$ of $\widetilde{G}^{s}$ can also be identified with

$$
\left\{x \in C^{+} \mid x^{6}+x=0,[x, U] \subset U\right\} .
$$

The spin representation $\rho^{s}$ of $\widetilde{G}^{s}$ and $\mathrm{g}^{s}$ is by definition the restriction on $\widetilde{G}^{s}$ and $\mathrm{g}^{S}$ of an absolutely irreducible representation $\phi$ of the associative algebra $C_{\boldsymbol{C}}^{+}$.

It is known that, for $a \in U$ with $Q(a)>0$, the correspondence $x \longmapsto$ $a^{-1} x^{c} a\left(x \in C^{+}\right)$is a positive involution of $C^{+}([3 \mathrm{~b}])$. Put

$$
\alpha_{0}: x \longmapsto \omega_{0}^{-1} x^{i} \omega_{0}
$$

and let $\mathscr{P}$ denote the cone of all positive elements in $C^{+}$with respect to this positive involution, i.e.,

$$
\mathscr{P}=\left\{b \in C^{+} \mid b^{\alpha} 0=b, \operatorname{tr}\left(x^{\alpha}{ }_{0} b x\right)>0 \text { for all } x \in C^{+}, x \neq 0\right\} .
$$

Then $\mathscr{P}$ is a self-dual homogeneous cone of classical type (the type depending on $m \bmod .8)$ and it is easy to see that $\omega \in \Omega$ implies $b=\omega_{0}^{-1} \omega \in \mathscr{P}$. The imbedding $\Omega \rightarrow \mathscr{P}$ thus defined is equivariant, for $\phi(g) \omega=g \omega g^{-1}$ goes to

$$
\omega_{0}^{-1} g \omega g^{-1}=\left(\omega_{0}^{-1} g \omega_{0}\right)\left(\omega_{0}^{-1} \omega\right) g^{-1}=\left(g^{\alpha} 0\right)^{-1} b g^{-1} .
$$


Clearly it is also linear. On the other hand, if one takes a basis of the representation-space $V$ such that one has $\psi\left(x^{\alpha} 0\right)={ }^{t} \overline{\psi(x)}$ for all $x \in C^{+}$, then every element in $\mathscr{P}$ is represented by a positive-definite hermitian matrix, and $\psi \mid \mathscr{P}$ is a linear imbedding as discussed above $\left(1^{\circ}-3^{\circ}\right)$. Therefore, the above imbedding $\Omega \rightarrow \mathscr{P}$, combined with $\phi$, gives a linear imbedding $\Omega \rightarrow \mathscr{P}(N, \boldsymbol{C}), N=\operatorname{dim} V$. Moreover, since the Cartan involution $\sigma=\sigma_{\omega_{0}}$ of $\mathrm{g}^{S}$ is given by $\sigma(x)=\omega_{0}^{-1} x \omega_{0}=-x^{\alpha}$, the condition $\left(C^{\prime}\right)$ is also satisfied. Thus, denoting by $F$ the standard hermitian form on $V$ with respect to the above basis, one obtains a strong solution $(V, \rho, F)$ for $\left(\mathfrak{g}, \omega_{0}\right)$.

Case $5^{\circ}$. One has $l=\operatorname{dim} \mathfrak{h}=6$ and $\operatorname{dim} \mathfrak{a}^{S}=2$. We enumerate the $\sigma$-fundamental system as shown in the diagram.

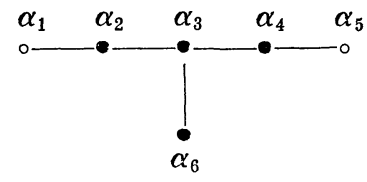

Then one has

$$
\mathfrak{a}^{s}=\left\{X \in \mathfrak{h}^{0} \mid \alpha_{i}(X)=0 \text { for } i=2,3,4,6\right\} .
$$

Put

$$
\gamma_{1}=\alpha_{1}\left|\mathfrak{a}^{s}, \quad \gamma_{2}=\alpha_{5}\right| \mathfrak{a}^{s}
$$

Then the restricted fundamental weights are given as follows (cf. [1], Planche V):

$$
\left\{\begin{array}{l}
\eta_{1}=\frac{4}{3} \gamma_{1}+\frac{2}{3} \gamma_{2}, \\
\eta_{2}=\frac{5}{3} \gamma_{1}+\frac{4}{3} \gamma_{2}, \\
\eta_{3}=2 r_{1}+2 \gamma_{2}, \\
\eta_{4}=\frac{4}{3} r_{1}+\frac{5}{3} r_{2}, \\
\eta_{5}=\frac{2}{3} \gamma_{1}+\frac{4}{3} \gamma_{2}, \\
\eta_{6}=\gamma_{1}+\gamma_{2} .
\end{array}\right.
$$

Since $\pi^{S}$ is the unique irreducible representation of $\mathrm{g}^{S}$ of dimension 27 , the highest (resp. lowest) weight of $\pi^{S}$ is $\widetilde{\omega}_{1}$ (resp. $-\widetilde{\omega}_{5}$ ), so that all restricted 
weights of $\pi^{s}$ are of the form

$$
\mu=-\frac{2}{3} r_{1}-\frac{4}{3} r_{2}+m_{1} r_{1}+m_{2} r_{2}, m_{1}, m_{2} \geqslant 0 .
$$

Hence by the condition $(L)$ one has

$$
\lambda_{\rho} s=\frac{1}{2}\left(\frac{2}{3}-m_{1}\right) r_{1}+\frac{1}{2}\left(\frac{4}{3}-m_{2}\right) r_{2} .
$$

But the above list shows that the coefficient of $\gamma_{1}$ in $\lambda_{\rho} s$ must be $\geqslant \frac{2}{3}$, which is impossible. Thus we have no solution in this case.

\section{Proof of Proposition 2.}

5.1. Let $(V, \rho, F)$ be a solution of our problem for $\left(g, \omega_{0}\right)$ (satisfying the conditions mentioned in 3.4). We consider the following two conditions for the representation $\rho^{S}$ :

$(K 1)$ For any irreducible subrepresentation $\rho_{1}^{S}$ of $\rho^{S}$, the restriction $\left.\rho_{1}^{S}\right|^{S}$ is irreducible.

(K2) For any two inequivalent irreducible subrepresentations $\rho_{1}^{s}$ and $\rho_{2}^{S}$ of $\rho^{S}, \rho_{1}^{S} \mid \mathfrak{f}^{S}$ and $\rho_{2}^{S} \mid \mathfrak{f}^{S}$ contain no irreducible representations of $\mathfrak{f}^{S}$ in common.

If the condition (K2) holds, then a primary component of $V$ with respect to $\rho^{s}$ is a direct sum of a certain number of primary components of $V$ with respect to $\rho^{s} \mid \mathfrak{f}^{S}$, which are mutually orthogonal. Hence one obtains the assertion (a) in Proposition 2. On the other hand, if the condition (K1) holds, then one obtains the assertion (c) in Proposition 2, whence follows immediately the assertion (b) (for a primary $\rho$ ) by an argument similar to that in [3a], p. 441. In view of the result of 4 , the only cases where the conditions $(K 1),(K 2)$ are violated are

(1) The case $4^{\circ}$ with $m$ even, where one has two spin representations $\rho_{1}^{S}$ and $\rho_{2}^{S}$ of $\mathrm{g}^{S}$, but $\rho_{1}^{S} \mid \mathfrak{f}^{S}$ and $\left.\rho_{2}^{S}\right|^{\mathfrak{f}^{S}}$ are irreducible and equivalent. Thus $\left(K_{2}\right)$ does not hold, if $\rho^{S}$ contains both $\rho_{1}^{S}$ and $\rho_{2}^{S}$.

(2) The case $4^{\circ}$ with $m$ odd, where one has only one spin representation $\rho_{1}^{s}$, but $\rho_{1}^{S} \mid \mathfrak{k}^{S}$ decomposes into the direct sum of two inequivalent irreducible representations of $\mathfrak{k}^{S}$. Thus $(K 1)$ does not hold.

Therefore, to complete the proof of Proposition 2, it is enough to show that the assertions (a) and (c) of Proposition 2 remain true even for these exceptional cases. 
5.2. The case $4^{\circ}, m$ : even. As is well-known, one has the direct decomposition

$$
C_{\boldsymbol{C}}^{+}=\mathfrak{\mho}_{1} \oplus \mathfrak{\mho}_{2}, \mathfrak{E}_{i} \cong M_{N}(\boldsymbol{C}) \quad\left(N=2^{m / 2-1}\right) .
$$

We fix an orthogonal basis $\left(e_{1}, \cdots, e_{m}\right)$ of $U$ such that $\omega_{0}=c e_{1}(c>0), Q\left(e_{1}\right)$ $=1, Q\left(e_{i}\right)=-1(2 \leqslant i \leqslant m)$. Put $\tilde{e}=e_{1} e_{2} \cdots e_{m}$. Then the unit element of $\mathfrak{F}_{i}$ is given by

$$
\varepsilon_{1}=\frac{1}{2}(1+\zeta \tilde{e}), \quad \varepsilon_{2}=\frac{1}{2}(1-\zeta \tilde{e}),
$$

where $\zeta$ is a 4 -th root of unity such that $\zeta^{2}=\tilde{e}^{2}=(-1)^{m / 2+1}$. Clearly the inner automorphism $x \longmapsto e_{1} x e_{1}$ of $C_{C}^{+}$interchanges the simple components $\mathfrak{E}_{1}$ and $\mathfrak{E}_{2}$, and leaves the subalgebra $C^{\prime+}$ of $C^{+}$generated by $e_{i} e_{j}(2 \leqslant i \leqslant j$ $\leqslant m$ ) elementwise invariant. Since one has $\bar{\varepsilon}_{1}^{t}=\varepsilon_{2}$, the correspondence $x \longmapsto$ $e_{1} \bar{x}^{t} e_{1}\left(x \in \mathfrak{E}_{i}\right)$ is a positive involution on each $\mathfrak{E}_{i}$. Therefore there exists an isomorphism $\psi_{1}: \mathfrak{C}_{1} \rightarrow M_{N}(\boldsymbol{C})$ such that one has

$$
\psi_{1}\left(e_{1} \bar{x}^{t} e_{1}\right)={ }^{t} \overline{\psi_{1}(x)} \quad \text { for all } x \in \mathfrak{c}_{1} .
$$

Then, the spin representations $\rho_{i}^{S}(i=1,2)$ (as matrix representations) are given as follows:

$$
\left\{\begin{array}{l}
\rho_{1}^{S}(g)=\phi_{1}\left(g \varepsilon_{1}\right) \\
\rho_{2}^{S}(g)=\phi_{1}\left(e_{1} g e_{1} \varepsilon_{1}\right)=\psi_{1}\left(e_{1} g \varepsilon_{2} e_{1}\right)
\end{array}\right.
$$

for $g \in \widetilde{G}^{s}$. In view of the relations $\bar{g}^{\imath}=g^{\ell}=g^{-1}, \bar{\varepsilon}_{1}^{\imath}=\varepsilon_{2}$, one obtains from (27) and (28) the relation

$$
\rho_{1}^{S}\left(g^{\alpha} 0\right)={ }^{t} \overline{\rho_{1}^{S}(g)}=\rho_{2}^{S}(g)^{-1} \quad \text { for } g \in \tilde{G}^{S} .
$$

In particular, for an element $k$ of the maximal compact subgroup $\widetilde{K}^{s}=$ $\tilde{G}^{s} \cap C^{++}$, one has

$$
\rho_{1}^{S}(k)=\rho_{2}^{S}(k) \text { : unitary. }
$$

Now let $(V, \rho, F)$ be any solution for $\left(g, \omega_{0}\right)$ and let $V=V^{(1)} \oplus V^{(2)}$ be the decomposition of $V$ into the direct sum of primary components such that $\rho^{(i)}=\rho \mid V^{(i)}$ contains $\rho_{i}^{S}$. If $V^{(1)}$ and $V^{(2)}$ were not orthogonal with respect to $F$, there would exist an irreducible subspace $V_{i}$ in $V^{(i)}$ for $i=1,2$, such that $V_{1}$ and $V_{2}$ are not orthogonal. For a suitable choice of basis in $V_{i}$, we may assume that the corresponding representations of $\tilde{G}^{s}$ on $V_{i}$ are 
given by (28). Then the matrix of $F \mid\left(V_{1}+V_{2}\right)$ would be of the form

$$
\left[\begin{array}{cc}
\alpha_{1} 1_{N} & \alpha_{12} 1_{N} \\
\bar{\alpha}_{12} 1_{N} & \alpha_{2} 1_{N}
\end{array}\right]
$$

with $\alpha_{1}, \alpha_{2}>0$ and $\alpha_{12} \neq 0$. Hence, for $g \in \tilde{G}^{s}$, one has

$$
\begin{aligned}
\rho\left(g\left(\omega_{0}\right)\right) \mid\left(V_{1}+V_{2}\right) & =\left[\begin{array}{cc}
\alpha_{1}\left(\rho_{1}(g)^{t} \overline{\rho_{1}(g)}\right)^{-1} & \alpha_{12}\left(\rho_{2}(g)^{t} \overline{\rho_{1}(g)}\right)^{-1} \\
\alpha_{12}\left(\rho_{1}(g)^{t} \overline{\rho_{2}(g)}\right)^{-1} & \alpha_{2}\left(\rho_{2}(g)^{t} \overline{\rho_{2}(g)}\right)^{-1}
\end{array}\right] \\
& =\left[\begin{array}{cc}
* & \alpha_{12} 1_{N} \\
\bar{\alpha}_{12} 1_{N} & *
\end{array}\right] .
\end{aligned}
$$

It would follow that there exists a linear function $\Phi$ on $U$ such that $\Phi(\omega)=1$ for all $\omega \in G^{S}\left(\omega_{0}\right)$, which is absurd.

5.3. The case $4^{\circ}, m$ : odd. We denote again by $C^{++}$the subalgebra of $C^{+}$generated by $e_{i} e_{j}(2 \leqslant i<j \leqslant m)$. Then one has

$$
\begin{aligned}
& C_{\boldsymbol{C}}^{+} \cong M_{N}(\boldsymbol{C}) \quad\left(N=2^{(m-1) / 2}\right), \\
& C_{\boldsymbol{C}}^{\prime+}=\widetilde{C}_{1}^{\prime} \oplus \widetilde{C}_{2}^{\prime}, \quad \widetilde{\Gamma}_{1}^{\prime} \cong \widetilde{C}_{2}^{\prime} \cong M_{N / 2}(\boldsymbol{C}) .
\end{aligned}
$$

We put $e_{-}=e_{2} \cdots e_{m}$. Then the unit element of $\mathfrak{C}_{i}^{\prime}(i=1,2)$ is given by

$$
\varepsilon_{1}^{\prime}=\frac{1}{2}\left(1+\zeta^{\prime} e_{-}\right), \quad \varepsilon_{2}^{\prime}=\frac{1}{2}\left(1-\zeta^{\prime} e_{-}\right),
$$

where $\zeta^{\prime}$ is a 4 -th root of unity such that $\zeta^{\prime 2}=e_{-}^{2}=(-1)^{(m-1) / 2}$. Again we fix an isomorphism $\phi: C_{C}^{+} \rightarrow M_{N}(\boldsymbol{C})$ such that

$$
\psi\left(e_{1} \bar{x}^{t} e_{1}\right)={ }^{t} \overline{\psi(x)} \quad \text { for all } x \in C_{\boldsymbol{C}}^{+} .
$$

Since one has $\overline{\zeta^{\prime} e_{-}}{ }^{\prime}=\zeta^{\prime} e_{-}, \phi\left(\varepsilon_{i}^{\prime}\right)$ is hermitian. Hence one may further assume that

$$
\psi\left(\varepsilon_{1}^{\prime}\right)=\left[\begin{array}{cc}
1_{N \prime} & 0 \\
0 & 0
\end{array}\right], \quad \psi\left(\varepsilon_{2}^{\prime}\right)=\left[\begin{array}{cc}
0 & 0 \\
0 & 1_{N^{\prime}}
\end{array}\right] \quad\left(N^{\prime}=N / 2\right) .
$$

The spin representation $\rho^{s}$ is given by the restriction of $\phi$ on $\tilde{G}^{s}$. Then $\rho^{s} \mid \tilde{K}^{s}$ is unitary and decomposes into the sum $\rho_{1}^{\prime s} \oplus \rho_{2}^{\prime s}$, where $\rho_{i}^{\prime s}$ is the spin representation of $\tilde{K}^{S}$ corresponding to the simple component $\mathbb{C}_{i}^{\prime}$.

Now let $(V, \rho, F)$ be an irreducible solution for $\left(\mathfrak{g}, \omega_{0}\right)$ and let $\dot{V}=V_{1}^{\prime}$ $\oplus V_{2}^{\prime}$ be the decomposition of $V$ into the direct sum of $\mathfrak{i}^{S}$-invariant subspaces $V_{i}^{\prime}$ corresponding to the representation $\rho_{i}^{\prime s}$. Then, in the basis 
chosen above, the matrix of $F$ is of the form

$$
\left[\begin{array}{cc}
\alpha_{1} 1_{N^{\prime}} & 0 \\
0 & \alpha_{2} 1_{N^{\prime}}
\end{array}\right]
$$

with $\alpha_{i}>0$. Therefore, to prove the assertion (c) of Proposition 2, it is enough to show that $\alpha_{1}=\alpha_{2}$. One has

$$
\begin{aligned}
\rho\left(\omega_{0}\right) & =\alpha_{1} \psi\left(\varepsilon_{1}^{\prime}\right)+\alpha_{2} \psi\left(\varepsilon_{2}^{\prime}\right) \\
& =\beta 1_{N}+\gamma \rho\left(e_{-}\right),
\end{aligned}
$$

where $\beta=\frac{1}{2}\left(\alpha_{1}+\alpha_{2}\right), \gamma=\frac{\zeta^{\prime}}{2}\left(\alpha_{1}-\alpha_{2}\right)$. Hence, for $g \in \tilde{G}^{s}$, one has

$$
\begin{aligned}
\rho\left(g\left(\omega_{0}\right)\right) & \left.={ }^{t} \overline{\rho(g}\right)^{-1}\left(\beta 1_{N}+\gamma \rho\left(e_{-}\right)\right) \rho(g)^{-1} \\
& =\rho\left(e_{1} g e_{1}\right)\left(\beta 1_{N}+\gamma \rho\left(e_{-}\right)\right) \rho(g)^{-1} \\
& =\beta \rho\left(e_{1} g e_{1} g^{-1}\right)+\gamma \rho\left(e_{-}\right),
\end{aligned}
$$

where $e_{1} g e_{1} g^{-1}$ is linear in $\omega=g\left(\omega_{0}\right)=g \omega_{0} g^{-1}$. Therefore, if $\gamma \neq 0$, there would again exist a linear function $\Phi$ on $U$ such that $\Phi(\omega)=1$ for all $\omega \in G^{S}\left(\omega_{0}\right)$, which is absurd.

\section{Real and quaternionic solutions.}

6.1. Let $V$ be a complex vector-space of dimension $n$. By a "real (resp. quaternion) structure" $J$ on $V$ we mean an antilinear transformation $J$ of $V$ such that $J^{2}=1$ (resp. -1 ). Given a real structure $J$ on $V$, the set $V_{\boldsymbol{R}}$ of all fixed elements of $J$ in $V$ becomes a real vector-space of dimension $n$ such that $V=V_{\boldsymbol{R}} \otimes_{\boldsymbol{R}} \boldsymbol{C}$. Given a quaternion structure $J$ on $V, n$ is necessarily even, and one can define a structure of a right $\boldsymbol{K}$-vector-space of dimension $\frac{n}{2}$ on $V$ by putting

$$
x j=J x \quad \text { for } x \in V,
$$

where as usual $\boldsymbol{K}$ is identified with $\boldsymbol{C}+\boldsymbol{C} \boldsymbol{j}$ by means of a fixed standard basis $(1, i, j, i j)$ of $\boldsymbol{K}$ over $\boldsymbol{R}$.

Now, let $(V, \rho, F)$ be a triple formed of a representation $(V, \rho)$ of $g$ and $F \in \mathscr{P}(V)$. If one has a real or quaternion structure $J$ on $V$ satisfying the following conditions

$$
\left\{\begin{aligned}
\rho(X) J & =J \rho(X) & & \text { for all } X \in \mathfrak{g}, \\
F(J x, J y) & =F(y, x) & & \text { for all } x, y \in V,
\end{aligned}\right.
$$


then we say that the triple $(V, \rho, F)$ admits a real or quaternion structure $J$, or $J$ is compatible with $(\rho, F)$. If $(V, \rho, F)$ admits a real structure $J$, then the real vector-space $V_{\boldsymbol{R}}$ defined above is $\rho(\mathfrak{g})$-invariant, and the restriction $\rho_{\boldsymbol{R}}$ of $\rho$ on $V_{\boldsymbol{R}}$ is a real representation $\rho_{\boldsymbol{R}}: \mathfrak{g} \rightarrow \mathfrak{g l}\left(V_{\boldsymbol{R}}\right)$. Moreover, $F(J x, y)$ $(x, y \in V)$ is bilinear and symmetric, so that, denoting by $S$ the restriction of $F$ on $V_{\boldsymbol{R}} \times V_{\boldsymbol{R}}$, one obtains an element $S \in \mathscr{P}\left(V_{\boldsymbol{R}}\right)$. Thus, if $(V, \rho, F)$ is a (complex) solution for $\left(\mathfrak{g}, \omega_{0}\right)$ admitting a real structure $J$, then the corresponding triple $\left(V_{\boldsymbol{R}}, \rho_{\boldsymbol{R}}, S\right)$ is a "real solution" for $\left(g, \omega_{0}\right)$ (i.e., a solution relative to $\left.\mathfrak{g}^{\prime}=\mathfrak{g} \mathfrak{l}(n, \boldsymbol{R})\right)$. Clearly, all real solutions are obtained in this way, and the equivalence classes of real solutions are in one-to-one correspondence with the equivalence classes of the quadruples $(V, \rho, F, J)$ defined in a natural manner.

Similarly, if a (complex) solution $(V, \rho, F)$ admits a quaternion structure $J$, then $(V / K, \rho)$ may be regarded as a quaternion representation $\rho: \mathfrak{g}$ $\rightarrow \mathfrak{g l}(V / \boldsymbol{K})$ and one can define a quaternion hermitian form $\boldsymbol{F}$ on $V$ by putting

$$
\boldsymbol{F}(x, y)=F(x, y)-F(x, J y) j \quad \text { for } x, y \in V .
$$

The triple $(V / \boldsymbol{K}, \rho, \boldsymbol{F})$ is then a "quaternionic solution" of our problem for $\left(\mathfrak{g}, \omega_{0}\right)$. All quaternionic solutions are obtained in this way, and the equivalence classes of quaternionic solutions are in one-to-one correspondence with the equivalence classes of the quadruples $(V, \rho, F, J)$ defined in a natural manner.

6. 2. We have seen above that the problem of finding all real or quaternionic solutions is equivalent to the determination of all complex solutions admitting a real or quaternion structure $J$. This latter problem can again be reduced to the irreducible case by the following Propositions.

Proposition 3. Let $(V, \rho, F)$ be a solution for $\left(g, \omega_{0}\right)$ and let

$$
V=V^{(1)} \oplus \cdots \oplus V^{(r)}
$$

be the direct decomposition of $V$ into primary components with respect to $\rho$. Put $\rho^{(i)}$ $=\rho\left|V^{(i)}, F^{(i)}=F\right| V^{(i)}$ and let $\rho_{i}$ be an irreducible representation of $g$ contained in $\rho^{(i)}$. Then $(V, \rho, F)$ admits a real (resp. quaternion) structure $J$, if and only if the following conditions are satisfied for all $1 \leqslant i \leqslant r$ :

(i) If $\bar{\rho}_{i} \sim \rho_{i}$, then the primary solution $\left(V^{(i)}, \rho^{(i)}, F^{(i)}\right)$ admits a real (resp. quaternion) structure. 
(ii) If $\bar{\rho}_{i}+\rho_{i}$, then there exists a (unique) index $i^{\prime}$ such that $\bar{\rho}_{i} \sim \rho_{i^{\prime}}$ and one has $\operatorname{dim} V^{(i)}=\operatorname{dim} V^{\left(i^{\prime}\right)}$. (In other words, $\rho_{i}$ and $\rho_{i^{\prime}}$ appear in $\rho$ with the same multiplicity.)

Suppose first that $(V, \rho, F)$ admits a real or quaternion structure $J$. Then $J$ induces a permutation of the $V^{(i)}$ s of order at most 2. If $J V^{(i)}=V^{(i)}$, then the primary solution $\left(V^{(i)}, \rho^{(i)}, F^{(i)}\right)$ admits a real or quaternion structure $J^{(i)}=J \mid V^{(i)}$. In this case, $\left(V^{(i)}, \rho^{(i)}, F^{(i)}\right)$ is self-conjugate, and one has $\bar{\rho}_{i} \sim \rho_{i}$. If $J V^{(i)}=V^{\left(i^{\prime}\right)}$ with $i \neq i^{\prime}$, two primary solutions $\left(V^{(i)}, \rho^{(i)}, F^{(i)}\right)$ and $\left(V^{\left(i^{\prime}\right)}, \rho^{\left(i^{\prime}\right)}, F^{\left(i^{\prime}\right)}\right.$ are conjugate to each other so that one has $\bar{\rho}_{i} \sim \rho_{i^{\prime}}$ and $\operatorname{dim} V^{(i)}=\operatorname{dim} V^{\left(i^{\prime}\right)}$. To prove the converse ("if" part), suppose the conditions (i), (ii) are satisfied. It is enough to show that, in the case (ii), the direct sum

$$
\left(V^{(i)} \oplus V^{\left(i^{\prime}\right)}, \quad \rho^{(i)} \oplus \rho^{\left(i^{\prime}\right)}, \quad F^{(i)} \oplus F^{\left(i^{\prime}\right)}\right)
$$

admits both real and quaternion structure. By the assumption, there exists a $\boldsymbol{C}$-antilinear isomorphism $\phi: V^{(i)} \rightarrow V^{\left(i^{\prime}\right)}$ such that one has

$$
\rho^{\left(i^{\prime}\right)}(X)=\phi \circ \rho^{(i)}(X) \circ \phi^{-1} \quad \text { for } X \in g .
$$

Put

$$
F^{\prime}(x, y)=F^{\left(i^{\prime}\right)}(\phi(y), \phi(x)) \quad \text { for } x, y \in V .
$$

Then the triple $\left(V^{(i)}, \rho^{(i)}, F^{\prime}\right)$ is a solution conjugate to $\left(V^{\left(i^{\prime}\right)}, \rho^{\left(i^{\prime}\right)}, F^{\left(i^{\prime}\right)}\right)$ and hence, by Theorem 2, it is equivalent to $\left(V^{(i)}, \rho^{(i)}, F^{(i)}\right)$. Therefore, by a suitable modification of $\phi$, we may assume that $F^{\prime}=F^{(i)}$. Then, putting

$$
J^{\left(i, i^{\prime}\right)}:\left(x, x^{\prime}\right) \longmapsto\left( \pm \phi^{-1}\left(x^{\prime}\right), \phi(x)\right) \quad\left(x \in V^{(i)}, x^{\prime} \in V^{\left(i^{\prime}\right)}\right),
$$

one obtains a real or quaternion structure (according to the sign \pm ) on $V^{(i)} \oplus V^{\left(i^{\prime}\right)}$ compatible with $\left(\rho^{(i)}+\rho^{\left(i^{\prime}\right)}, F^{(i)}+F^{\left(i^{\prime}\right)}\right)$. (Note that the equivalence class of such a real or quaternion structure is uniquely determined, independently of the choice of $\phi$.)

Proposition 4. The notation being as in Theorem 1, (b), suppose that a primary solution $(V, \rho, F)$ admits a real (resp. quaternion) structure $J$. Then, $J$ can be written uniquely in the form

$$
J=J_{1} \otimes J_{0},
$$

where $J_{1}$ is a real or quaternion structure on $V_{1}$ compatible with $\left(\rho_{1}, F_{1}\right)$ and $J_{0}$ is a real or quaternion (resp. quaternion or real) structure on $V_{0}$ compatible with 
$\left(\rho_{0}, F_{0}\right)$. Conversely, an antilinear automorphism $J$ of $V$ defined in this way is a real (resp. quaternion) structure on $V$ compatible with $(\rho, F)$.

This follows immediately from Schur's lemma and the uniqueness of $F_{1}$ (Prop. 2, (c)). Note that the condition (35) for $J_{0}$ means that the representation $\rho_{0}$ is essentially a representation of $\mathfrak{f}_{0} \oplus \mathfrak{a}_{0}$ into $\mathfrak{p}\left(n_{0}, \boldsymbol{R}\right)$ (the Lie algebra of all $n_{0} \times n_{0}$ real skew-symmetric matrices) or $\mathfrak{H}\left(\frac{n_{0}}{2}, \boldsymbol{K}\right)$ (the Lie algebra of all $\frac{n_{0}}{2} \times \frac{n_{0}}{2}$ quaternion skew-hermitian matrices), where $n_{0}=$ $\operatorname{dim}_{c} V_{0}$.

6. 3. It remains to check for each (self-conjugate) irreducible solution given in 4 whether or not it admits a real or quaternion structure. Note that, if an irreducible solution admits a real (resp. quaternion) solution, then such a structure is unique, and the solution does not admit any quaternion (resp. real) structure.

Case $1^{\circ}$. The unique irreducible solution clearly admits a real structure.

Case $2^{\circ}$. The two irreducible solutions are conjugate to each other, so that neither of them admits a real or quaternion structure.

Case $3^{\circ}$. The unique irreducible solution clearly admits a quaternion structure.

Case $4^{\circ}, m$ : even. It is known that

$$
C^{+} \cong \begin{cases}M_{N}(\boldsymbol{C}) & \text { if } m \equiv 0(\bmod .4), \\ M_{N}(\boldsymbol{R}) \oplus M_{N}(\boldsymbol{R}) & \text { if } m \equiv 2(\bmod .8), \\ M_{N / 2}(\boldsymbol{K}) \oplus M_{N / 2}(\boldsymbol{K}) & \text { if } m \equiv 6(\bmod .8),\end{cases}
$$

where $N=2^{m / 2-1}$. Therefore, if $m \equiv 0(\bmod .4)$, the two irreducible solutions are not self-conjugate, admitting no real or quaternion structure. If $m \equiv 2$ (mod. 4), then from what we mentioned in 4 and 5 each of the two irreducible solutions admits a real or quaternion structure, according as $m \equiv$ 2 or $6(\bmod .8)$.

Case $4^{\circ}, m:$ odd. One has

$$
C^{+} \cong \begin{cases}M_{N}(\boldsymbol{R}) & \text { if } m \equiv 1,3(\bmod .8), \\ M_{N / 2}(\boldsymbol{K}) & \text { if } m \equiv 5,7(\bmod .8),\end{cases}
$$

where $N=2^{(m-1) / 2}$. Therefore, the unique irreducible solution admits a real or quaternion structure according as $m \equiv 1,3$ or 5,7 (mod. 8 ). 


\section{BIBLIOGRAPHY}

[ 1 ] Bourbaki, N. Groupes et algèbres de Lie, Chap. 4, 5 et 6, Eléments de Math., Hermann, Paris, 1968.

[2]] Koecher, M. (a) Positivitätsbereich in $R^{n}$, Amer. J. of Math. 79 (1957), 575-596.

(b) Analysis in reellen Jordan Algebren, Nachr. Akad. Wiss. Göttingen Math-Phys.

Kl. IIa (1958), 67-74.

(c) Die Geodätischen von Positivitätsbereichen, Math. Ann. 135 (1958), 192-202.

[3] Satake, I. (a) Holomorphic imbeddings of symmetric domains into a Siegel space, Amer. J. of Math. 87 (1965), 425-461.

(b) Clifford algebras and families of abelian varieties, Nagoya Math. J. 27 (1966), 435 -446; Corrections, ibid. 31 (1968), 295-296.

(c) Symplectic representations of algebraic groups satisfying a certain analyticity condition, Acta Math. 117 (1967), 425-461.

[4] Vinberg, E.B. (a) Homogeneous cones, Dokl. Akad. Nauk SSSR 133 (1960), 9-12 (=Soviet Math. Dokl. 1 (1961), 787-790).

(b) The theory of homogeneous convex cones, Trudy Moskov. Mat. Obšč. 12 (1963), 303-358 (=Trans. of Moscow Math. Soc. 1963, 340-403).

University of California, Berkely 